\title{
A Deeply Buried Narrow-line Seyfert 1 Nucleus Uncovered in Scattered Light
}

\author{
Xiang Pan ${ }^{1,2}$ (1), Honglin $\mathrm{Lu}^{2}$, S. Komossa ${ }^{3}$ (1), Dawei Xu ${ }^{4}$, Weimin Yuan ${ }^{4}$, Luming Sun ${ }^{2}$, Paul S. Smith ${ }^{5}$, Shaohua Zhang ${ }^{1}$ (D), \\ Peng Jiang $^{1}{ }_{1}$, Chenwei Yang ${ }^{1}$, Wenjuan Liu ${ }^{6}$, Ning Jiang ${ }^{3}$ (iD), Y. E. Rashed ${ }^{7}$, A. Eckart ${ }^{8}$, Jens Dierkes ${ }^{8}$, and Hongyan Zhou ${ }^{1,2}$ (i) \\ ${ }^{1}$ Polar Research Institute of China, 451 Jinqiao Road, Shanghai, 200136, People's Republic of China; zhouhongyan@ pric.org.cn \\ ${ }^{2}$ Key Laboratory for Researches in Galaxies and Cosmology, Department of Astronomy, University of Sciences and Technology of China, Chinese Academy of \\ Sciences, Hefei, Anhui, 230026, People's Republic of China \\ ${ }^{3}$ Max Planck Institut für Radioastronomie, Auf dem Hügel 69, D-53121 Bonn, Germany \\ ${ }^{4}$ National Astronomical Observatories, Chinese Academy of Sciences, Beijing 100012, People's Republic of China \\ ${ }^{5}$ Steward Observatory, University of Arizona, Tucson, AZ 85721, USA \\ ${ }^{6}$ Yunnan Observatories, Chinese Academy of Sciences, Kunming, Yunnan 650011, People's Republic of China \\ ${ }_{7}$ Department of Astronomy, Faculty of Science, University of Baghdad, 10071 Baghdad-Aljadirya, Iraq \\ ${ }^{8}$ University and City Library Cologne, Department Research and Publication Support, University of Cologne, Universitätsstr, 33, D-50931 Cologne, Germany \\ Received 2018 May 30; revised 2018 November 9; accepted 2018 November 15; published 2019 January 10
}

\begin{abstract}
We present spectropolarimetric and spectrophotometric observations of the peculiar active galactic nucleus (AGN) SDSS J120300.19+162443.7 (hereafter J1203+1624) at $z=0.1656$. Its optical total flux spectra clearly show broad emission lines (BELs) in $\mathrm{H} \alpha$ and $\mathrm{H} \beta$. After removal of narrow emission lines (NELs), the full width at half maximum (FWHM) of the Lorentzian BEL is FWHM $\mathrm{BEL} \approx 1270 \mathrm{~km} \mathrm{~s}^{-1}$, fulfilling the conventional definition of a narrow-line Seyfert 1 (NLS1) galaxy. However, its NELs are unprecedentedly strong when compared to type 1 AGNs. This, together with its large MIR excess $\left(g-W_{4}=13.172 \mathrm{mag}\right)$, implies that the observer and the NEL region might see a different ionization continuum. Our optical spectropolarimetry confirms its type 2 nature by detecting a polarized blue continuum and Balmer BELs $\left(F W H M_{\text {Polarized BEL }} \approx 1183 \mathrm{~km} \mathrm{~s}^{-1}\right.$ ), with a high polarization degree of $>20 \%$ in the blue wing. The mass and Eddington rate of the central black hole are estimated based on both transmitted and scattered AGN radiation, which is $M_{0}<2.9 \times 10^{7} M_{\odot}$ and $L_{\mathrm{bol}} / L_{\mathrm{Edd}}>1.5$. Severe extinction of the AGN emission also enables a clear view of the compact host galaxy. The discovery of J1203 +1624 suggests that NLS1 follows the AGN unification scheme, and studying its analogs could blaze a new trail for exploring the connection between black hole growth and star formation in the host galaxy. The interesting features of J1203+1624, like the peculiar NELs and inflowing scattering clouds within the sublimation radius, are worth detailed follow-ups in the future.
\end{abstract}

Key words: galaxies: Seyfert - quasars: emission lines - quasars: individual (SDSS J120300.19+162443.7)

\section{Introduction}

In the 1990s, an orientation-based unified model was proposed that explains the spectroscopic classification of active galactic nuclei (AGNs; Antonucci 1993). In this toy model, an AGN consists of an accretion disk near the black hole, a fastrotating broad emission-line region (BELR), and a more distant narrow emission-line region (NELR) in the ionization cone that produce the continuum, broad emission lines (BELs), and narrow emission lines (NELs), respectively. If the observers line of sight lies within the ionization cone, then a type 1 AGN is observed where the disk continuum and the emission from the BLR and NLR are all in direct view. A dusty torus is also assumed that, if viewed edge-on, will block the disk continuum and BELR in our line of sight, giving rise to "type 2" AGNs such as a Seyfert 2 galaxy (Peterson 1997).

The term narrow-line Seyfert 1 (NLS1) galaxy was initially denominated by Osterbrock \& Pogge (1985) to categorize an "amazing" class of AGN (Tarchi et al. 2011) that shows a prominent disk continuum, but their broad line-emission widths are much narrower than those for normal Seyfert 1 nuclei (BLS1s). There are other observational differences between NLS1s and BLS1s, including that (1) the Fe II emission lines in NLS1s are often stronger, (2) the BEL profile of NLS1s is often better described by a Lorentzian than a Gaussian function (e.g., Véron-Cetty et al. 2001; Zhou et al. 2006), (3) NLS1s more frequently show a steep photon index and short-timescale/large-amplitude variability in the X-rays (e.g., Leighly 1999a, 1999b), and (4) the fraction of radioloud NLS1s is much less than that of BLS1s (Zhou et al. 2002; Komossa et al. 2006; Zhou et al. 2006; Yuan et al. 2008). It is now generally thought that these extreme properties of NLS1s result from smaller supermassive black holes (SMBHs) accreting at higher Eddington rates than BLS1s.

On the other hand, it has been proposed that the narrower BELs in NLS1s come of from disk-like BELR viewed at a small inclination angle from face-on (Osterbrock \& Pogge 1985; Leighly 1999b; Komossa et al. 2006). This is supported by the fact that at smaller inclinations, the observed emissionline widths of quasars are relatively smaller (e.g., Wills \& Browne 1986; Rokaki et al. 2003). Also, the absence of absorption by materials of the BELR in type 1 quasars (Antonucci et al. 1989) and the modeling of the velocityresolved reverberation mapping results (e.g., Grier et al. 2013; $\mathrm{Li}$ et al. 2013) both favor a disk-like BELR geometry. However, this geometrical interpretation alone cannot explain all of the extreme properties of NLS1s (Reviewed in Komossa 2008). This is supported by the spectropolarimetric study of the orientation of NLS1s (Robinson et al. 2011). Nevertheless, both our current samples and interpretations of NLS1s prefer small inclination angles, from which the continuum and BELs are too strong for analysis of weak structures like NELRs, host galaxies, etc. If NLS1s follow the "strong" unification model of AGNs, there should be its type 2 counterparts (NLS2s), which are viewed nearly edge-on. Studying them may reveal hidden 
properties that can be crucial in understanding AGNs with extreme properties, such as NLS1s.

There are studies devoted to identifying NLS1s along AGN unification sequences, including NLS2s and intermediate-type counterparts of NLS1s (NLS1.8s or 1.9s; Osterbrock 1981). Zhang et al. (2017b) recently presented a detailed analysis of three optically classified NLS1s with $E_{B-V} \sim 1$ mag reddened by dusty absorbers at torus scales. As extinction grows stronger, BELs can only be detected in the IR because of relatively less extinction at longer wavelengths. The $\mathrm{Pa} \beta$ BELs of FWHM $<2000 \mathrm{~km} \mathrm{~s}^{-1}$ are reported in two optically obscured Seyfert galaxies, NGC 5506 (Nagar et al. 2002) and Mrk 573 (Ramos Almeida et al. 2008), making them good candidates for moderately buried NLS1s. However, in type 2 AGNs, where dust extinction is much stronger, transmitted BELs are so weak that measurements of line width and verification of their NLS1 nature becomes difficult. Dewangan \& Griffiths (2005) reported three type 1.9/2 Seyfert galaxies with NLS1-like X-ray properties and suggested them as NLS2s. Based on either IR BELs or X-ray properties, these candidates are identified differently from the conventional NLS1s, and their hidden BELs cannot be easily probed. In this paper, we report the discovery of a bona fide NLS2, SDSS J120300.19+162443.7 (hereafter J1203+1624). We identified it as an NLS1 based on its total flux optical spectrum, while its type 2 nature is implied by its superstrong NELs and extremely high flux excess in the FIR. Our follow-up optical spectropolarimetry revealed the scattered BEL component and confirmed its NLS2 nature.

\section{Observations}

With an archival SDSS (York et al. 2000) spectrum (3100-9180 ^) observed on 2007 April 17, J1203+1624 is identified as an NLS1 in our systematic study of SDSS quasars (Section 3). This spectrum is combined with the UV spectrum obtained on 2006 March 28 by GALEX (Morrissey et al. 2007), and broadband photometry from GALEX (UV), SDSS (optical), the Two Micron All Sky Survey (2MASS, near-IR; Skrutskie et al. 2006), and the Wide-field Infrared Survey Explorer (WISE, mid-IR; Wright et al. 2010) is collected to study the spectral energy distribution (SED) of J1203+1624.

The strong NELs are very unusual for an NLS1. We followed up by observing it using the $6.5 \mathrm{~m}$ MMT with the blue channel spectrograph (Schmidt et al. 1989) on 2011 December 27 (G800 grating, [3350-5100] $\AA$ ) and 2012 February 29 (G600 grating, [8770-9520] $\AA$ ). We also performed optical spectroscopy of J1203+1624 with the MODS spectrograph on the Large Binocular Telescope (LBT; ${ }^{9} 3200-10200 \AA$ ) on 2012 February 01 (Rashed et al. 2015). The SDSS, MMT/BC, and LBT/MODS spectra are in similar resolutions. After scaling the three spectra at a similar continuum flux level, no obvious variability in their spectral shape is found. This is consistent with the stable $V$-band magnitude of $18.10 \pm 0.11$ monitored by the Catalina Sky Survey ${ }^{10}$ between 2005 April 9 and 2013

\footnotetext{
9 The LBT is an international collaboration among institutions in the United States, Italy, and Germany. The LBT Corporation partners are the University of Arizona, on behalf of the Arizona Board of Regents; Istituto Nazionale di Astrofisica, Italy; LBT Beteiligungsgesellschaft, Germany, representing the Max Planck Society, the Leibniz Institute for Astrophysics Potsdam, and Heidelberg University; The Ohio State University; and the Research Corporation, on behalf of the University of Notre Dame, University of Minnesota, and University of Virginia.

10 http://nesssi.cacr.caltech.edu/DataRelease/
}

June 11 (Catalina ID: CSS_J120300.2+162444). In addition, a medium-resolution NIR spectrum (9200-25000 А) was obtained on 2013 February 23 with the TripleSpec spectrograph on the Palomar 200 inch Hale telescope, which helps diagnose its emission-line systems. And optical spectropolarimetry was carried out with MMT/SPOL (Schmidt et al. 1992) on 2016 April 5 to search for scattered emission in the optical band. With a $600 \mathrm{~g} \mathrm{~mm}^{-1}$ grating, the SPOL data cover a wavelength range of $[4200,8200] \AA$. Since variability is not detected, and the optical-IR spectra of J1203+1624 have similar resolutions, they are combined to reach a high signal-tonoise ratio and wide wavelength coverage. All data are corrected for the Galactic extinction of $E_{B-V}=0.034$ according to the dust map in Schlafly \& Finkbeiner (2011) and deredshifted with $z=0.16559$ determined by the NELs before follow-up analysis. Throughout this paper, we adopt a cosmological model with parameters $H_{0}=70 \mathrm{~km} \mathrm{~s}^{-1} \mathrm{Mpc}^{-1}$, $\Omega_{\mathrm{M}}=0.3$, and $\Omega_{\Lambda}=0.7$.

\section{An Unusual NLS1}

Following Zhou et al. (2006), we carry out an iterative approach to fit both the continuum and emission lines of the optical spectrum of $\mathrm{J} 1203+1624$. Here is a brief summary. In step 1 , the optical continuum is fitted in continuum windows with a combination of host starlight and nuclear emission. The simple stellar population (SSP) models in Bruzual \& Charlot (2003) are assumed as host starlight, reddened with a Milky Way extinction law (Fitzpatrick \& Massa 2007). The nuclear emission includes a power-law (PL) continuum and a twocomponent analytic Fe II emission model (Dong et al. 2005) that incorporates the VJV04 Fe II template (Véron-Cetty et al. 2004). During continuum fitting, spectral regions around strong stellar absorption features (gray regions in the lower panel of Figure 1) are weighted higher than the rest of the data points so as to ensure a reasonable fit to the SSP component. The fitted continuum model is then subtracted, and the line spectrum is obtained. In step 2, the line spectrum derived in the first step is fitted. Besides the prominent NELs, a broad base is clearly seen at $\mathrm{H} \alpha$, indicating the presence of a (low-ionization) $\mathrm{BEL}$ component. A test fitting of the $\mathrm{H} \alpha+[\mathrm{N} \mathrm{II}]+[\mathrm{S} \mathrm{II}]$ complex shows that the NELs are in the form of a single Gaussian, while a Lorentzian BEL can fit the data better than a Gaussian BEL. Thus, each of the identified NELs/BELs in the line spectrum are assigned a Gaussian/Lorentzian profile, with their line centroids and widths bound together in velocity space. Then the line spectrum is modeled. In step 3 , we repeat the fitting of the continuum and emission lines until the model parameters converge. The converged continuum and emission-line models are displayed in Figure 1. Finally, we resampled the spectral data and carried out the fitting 1000 times with a bootstrap approach. The results are then compared in obtaining uncertainties of model parameters. The modeled fluxes of each NEL/BEL are listed in Table 1.

The spectral modeling reveals a blue nucleus with a PL at $\alpha_{\lambda}=-1.36 \pm 0.24$. The widths of the Lorentzian BELs are FWHM $_{\text {BEL }}=1270 \pm 18 \mathrm{~km} \mathrm{~s}^{-1}$, four times the width of the Gaussian NELs of FWHM $_{\text {NEL }}=305 \pm 1 \mathrm{~km} \mathrm{~s}^{-1}$, making the narrow- and broad-line components relatively easy to separate. Thus, J1203+1624 fulfills the criterion of FWHM $_{\text {BEL }}<2200 \mathrm{~km} \mathrm{~s}^{-1}$ for NLS1s (Zhou et al. 2006; Gelbord et al. 2009). A Balmer flux ratio of the Lorentzian BEL $F_{\mathrm{H} \alpha \text { BEL }} / F_{\mathrm{H} \beta \text { BEL }}=2.79 \pm 0.43$ is found, consistent with 


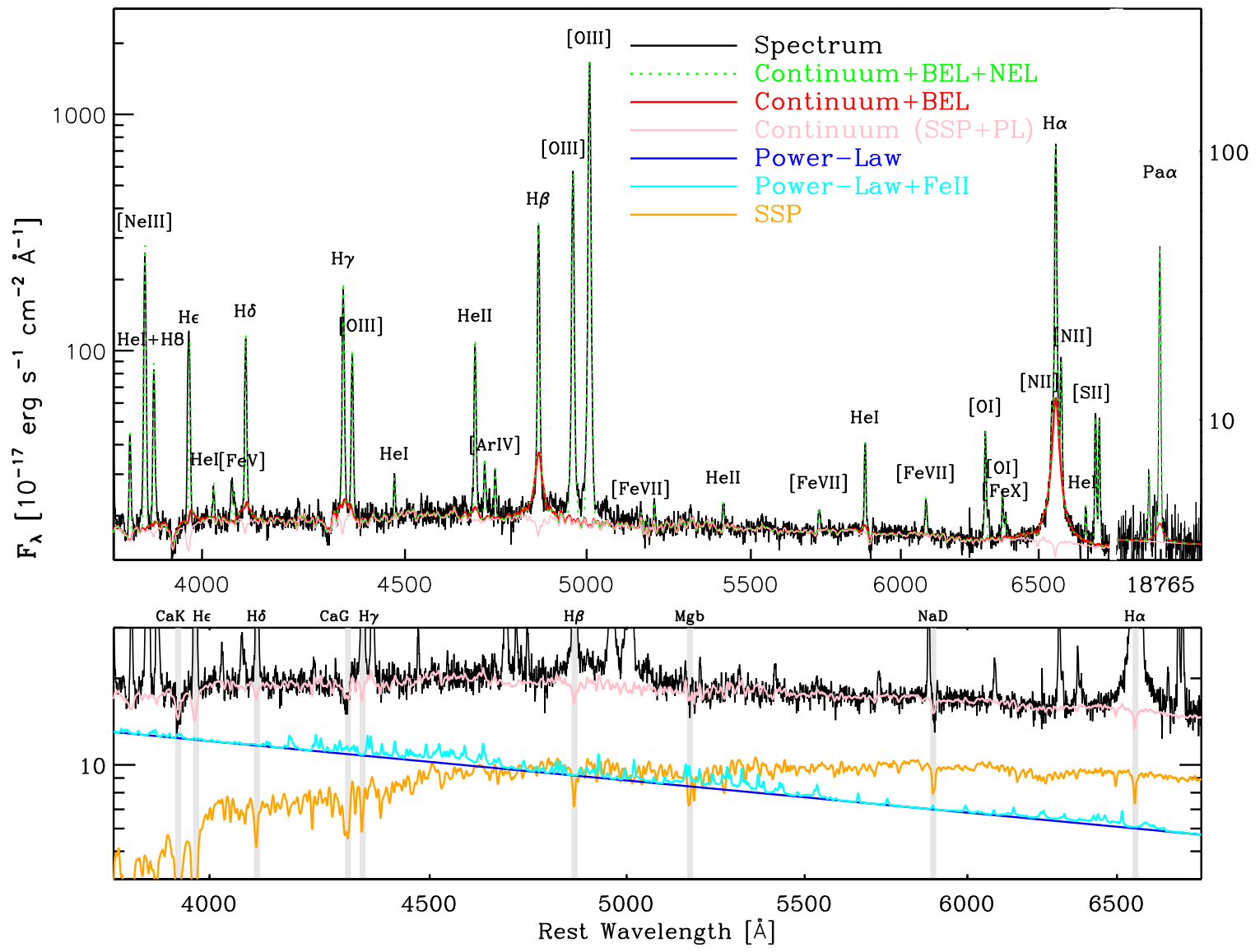

Figure 1. Spectral modeling of J1203+1624. The observed data are shown with black lines. The best-fit models, i.e., continuum, continuum+BEL, and continuum + BEL+NEL, are overlaid in different colors in the upper panel. In the lower panel, a zoom-in comparison between the data and modeled continuum (PL+Fe II +SSP) components is shown, with prominent stellar absorption lines marked with vertical gray lines.

the sample mean of $3.028 \pm 0.017$ for NLS1s (Zhou et al. 2006). Another typical component for NLS1s, Fe II emission, is marginally detected in $\mathrm{J} 1203+1624$, with a relative intensity $R_{4570} \equiv F_{\mathrm{Fe} \text { II }} \lambda \lambda 4344-4684 / F_{\mathrm{H} \beta}=0.25 \pm 0.05$. The total flux ratio [O III] $\lambda 5007 / \mathrm{H} \beta$ is found to be $4.45 \pm 0.26$, which exceeds the upper limit of 3 adopted for NLS1s. In early works, the [O III] $\lambda 5007 / \mathrm{H} \beta<3$ criterion is to ensure the existence of a Balmer BEL system in the nuclei of NLS1 candidates. The BEL system in J1203+1624 is directly observed, and the detection of Fe II multiplets and high-ionization [Fe VII], [Fe X] emission lines suggests the presence of the AGN. Therefore, this line ratio diagnosis is not prerequisite (Pogge 2000; Zhou et al. 2006), and J1203+1624 is identified as an NLS1 by any standard.

As shown in Figure 2, the Balmer decrements of NELs in $\mathrm{J} 1203+1624$ are found to be in excellent agreement with that of the Case-B prediction (Osterbrock \& Ferland 2006), which means negligible dust extinction to the NELR in J1203. We then checked J1203+1624 in the BPT diagrams (Figure 3) for an empirical diagnosis of the NELs. Given the presence of various strong high-ionization lines, the NEL of J1203+1624 is expected to be AGN-dominated. However, J1203+1624 is unexpectedly diagnosed as a composite of AGNs and starforming ([H II]) regions. With a similar $[\mathrm{O} \mathrm{III]} / \mathrm{H} \alpha$ ratio to AGNs, the ionization source of the NELR can be AGNdominated in $\mathrm{J} 1203+1624$. On the other hand, the relatively low $[\mathrm{N} \mathrm{II}] / \mathrm{H} \alpha$ ratio may indicate a relatively low metallicity of the NELR. The most unusual property of the NELs in $\mathrm{J} 1203+1624$ as an NLS1, though, is the high intensity. At $\mathrm{EW}_{\text {[O III] } \lambda 5007}=583 \pm 16 \AA$, it is even higher than $97 \%$ of the SDSS DR1 type 2 quasars (Zakamska et al. 2003). The question is raised as to whether or not J1203+1624 is a Seyfert 2 galaxy. We thus check the SED of J1203+1624 in a broader wavelength range for additional clues in Section 4.

\section{Obscured and Scattered Nuclear Emission}

\subsection{The Deeply Buried Nucleus}

As shown in Figure 4, the GALEX data in the UV $(\lesssim 2500$ $\AA$ in the quasar's rest frame) appear to be the natural extent of the nuclear emission model described in Section 3, while the MIR-to-optical color of $\mathrm{J} 1203+1624$ is $g-W_{4}=13.172 \pm$ $0.050 \mathrm{mag}, 2.3 \mathrm{mag}$ higher than the quasar composite, and indicates a significant flux excess in the MIR. A similar MIR flux excess is found in the quasar SDSS J000610.67+121501.2 (SDSS J0006+1215), which turns out to be heavily obscured AGN radiation (Zhang et al. 2017a).

We then modeled the UV-optical-IR SED of J1203+1624 with three components, including (1) a blue nuclear emission $\left(B_{\lambda}\right)$ that dominates in the UV, (2) a reddened AGN emission $\left(R_{\lambda}\right)$ as indicated by the MIR flux excess, and (3) starlight from the host galaxy $\left(H_{\lambda}\right)$, which contributes significantly in the optical and NIR (Section 3). Since the spectral model of the blue nucleus obtained in Section 3 is similar to the quasar 
Table 1

Emission-line Intensities

\begin{tabular}{|c|c|c|}
\hline ID & $\lambda_{\mathrm{lab}}{ }^{\mathrm{a}}$ & $L / 10^{40} \mathrm{erg} \mathrm{s}^{-}$ \\
\hline \multicolumn{3}{|l|}{$\mathrm{NELs}^{\mathrm{b}}$} \\
\hline H9 & 3836.47 & $6.8 \pm 0.3$ \\
\hline [Ne III] & 3869.85 & $60.9 \pm 1.0$ \\
\hline $\mathrm{He} \mathrm{I}$ & 3889.74 & $13.1 \pm 4.2$ \\
\hline H8 & 3890.15 & $3.8 \pm 4.0$ \\
\hline $\mathrm{H} \epsilon$ & 3971.20 & $21.5 \pm 2.8$ \\
\hline $\mathrm{He} \mathrm{I}+\mathrm{N}$ II & 4027.28 & $2.0 \pm 0.4$ \\
\hline$[\mathrm{Fe} \mathrm{v}]$ & 4072.39 & $2.1 \pm 0.1$ \\
\hline $\mathrm{H} \delta$ & 4102.89 & $22.6 \pm 2.8$ \\
\hline $\mathrm{H} \gamma$ & 4341.68 & $45.1 \pm 3.1$ \\
\hline [O III] & 4364.44 & $21.3 \pm 0.4$ \\
\hline He I & 4472.76 & $2.8 \pm 0.7$ \\
\hline He II & 4687.02 & $25.2 \pm 0.6$ \\
\hline $\mathrm{He} \mathrm{I}$ & 4713.15 & $4.6 \pm 0.6$ \\
\hline [Ar IV] & 4741.50 & $3.6 \pm 0.4$ \\
\hline $\mathrm{H} \beta$ & 4862.68 & $88.6 \pm 3.5$ \\
\hline [O III] & 4960.29 & $162.5 \pm 16.5$ \\
\hline [O III] & 5008.24 & $492.2 \pm 50.1$ \\
\hline [Fe VII] & 5160.33 & $0.5 \pm 0.3$ \\
\hline$[\mathrm{Fe} \mathrm{VI}]$ & 5177.48 & $0.5 \pm 0.1$ \\
\hline$[\mathrm{N} \mathrm{I}]$ & 5200.53 & $1.4 \pm 0.2$ \\
\hline [Fe VII $]$ & 5277.85 & $0.0 \pm 0.1$ \\
\hline [Fe XIV] & 5304.34 & $0.4 \pm 0.1$ \\
\hline He II & 5413.60 & $1.7 \pm 0.4$ \\
\hline$[\mathrm{Fe}$ VII $]$ & 5722.30 & $1.6 \pm 0.2$ \\
\hline $\mathrm{He} \mathrm{I}$ & 5877.29 & $8.4 \pm 0.4$ \\
\hline [Fe VII] & 6087.98 & $2.7 \pm 0.2$ \\
\hline O I & 6302.05 & $11.6 \pm 0.5$ \\
\hline O I & 6365.54 & $3.6 \pm 0.2$ \\
\hline$[\mathrm{Fe} \mathrm{X}]$ & 6376.30 & $1.4 \pm 0.2$ \\
\hline [N II] & 6549.85 & $8.2 \pm 0.4$ \\
\hline $\mathrm{H} \alpha$ & 6564.61 & $266.8 \pm 6.1$ \\
\hline$[\mathrm{N}$ II] & 6585.28 & $24.7 \pm 1.1$ \\
\hline $\mathrm{He} \mathrm{I}$ & 6679.70 & $2.4 \pm 0.2$ \\
\hline [S II] & 6718.29 & $15.3 \pm 0.4$ \\
\hline [S II] & 6732.67 & $14.5 \pm 0.4$ \\
\hline \multicolumn{3}{|l|}{$\mathrm{BELs}^{\mathrm{c}}$} \\
\hline $\mathrm{H} 8$ & 3890.15 & $0.5 \pm 1.2$ \\
\hline $\mathrm{H} \epsilon$ & 3971.20 & $3.8 \pm 2.6$ \\
\hline $\mathrm{H} \delta$ & 4102.89 & $7.5 \pm 3.3$ \\
\hline $\mathrm{H} \gamma$ & 4341.68 & $4.6 \pm 5.8$ \\
\hline $\mathrm{He}$ II & 4687.02 & $4.1 \pm 1.9$ \\
\hline $\mathrm{H} \beta$ & 4862.68 & $40.1 \pm 3.4$ \\
\hline $\mathrm{He} \mathrm{I}$ & 5877.29 & $1.8 \pm 0.8$ \\
\hline $\mathrm{H} \alpha$ & 6564.61 & $112.6 \pm 9.7$ \\
\hline
\end{tabular}

Notes.

${ }^{\text {a }}$ References for the identified emission lines are Liu et al. (2000) and Vanden Berk et al. (2001).

${ }^{\mathrm{b}}$ NELs are in the profile of a Gaussian, with a width of $\mathrm{FWHM}_{\mathrm{NEL}}=305 \pm$ $1 \mathrm{~km} \mathrm{~s}^{-1}$.

${ }^{c}$ BELS are in the profile of a Lorentzian, with a width of $\mathrm{FWHM}_{\mathrm{BEL}}=1270 \pm 18 \mathrm{~km} \mathrm{~s}^{-1}$.

composite in both their spectral indices and Balmer decrements, we follow Zhou et al. (2010) in applying the broadband composite quasar spectrum $\left(Q_{\lambda}\right)$ as blue nuclear emission $B_{\lambda}=a Q_{\lambda}$, which largely simplifies our SED models. We assume $R_{\lambda}=b 10^{-0.4 A_{\lambda}^{\text {nucleus }}} Q_{\lambda}$ as the reddened AGN emission. Since a steep extinction law is favored for AGNs from recent

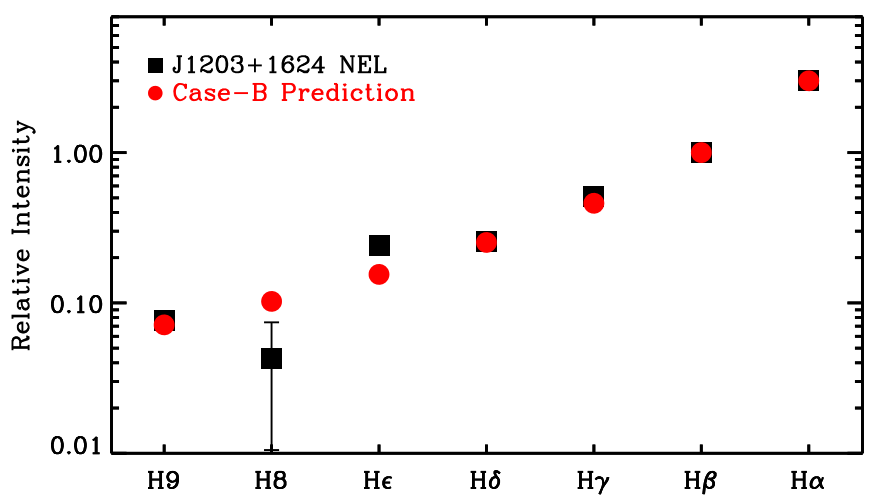

Figure 2. Excellent agreement is found between the Balmer decrements of NELs in J1203+1624 and the Case-B model prediction, which indicates negligible dust extinction to the NELR.

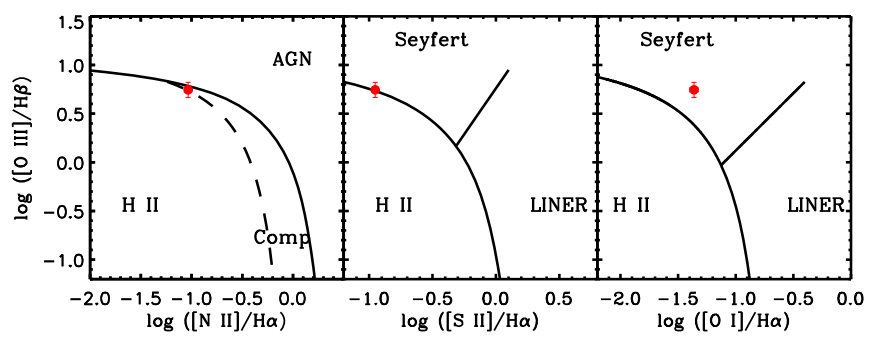

Figure 3. NEL ratios of $\mathrm{J} 1203+1624$ in the BPT diagrams (Baldwin et al. 1981; Kewley et al. 2001; Kauffmann et al. 2003), which is diagnosed as a composite of AGN and star-forming ([H II]) regions.

observations (e.g., Jiang et al. 2013; Zafar et al. 2015), the extinction law for the Small Magellanic Cloud (SMC; Martin et al. 1989) is adopted as $A_{\lambda}^{\text {nucleus }}$. Similar to Section 3, for the stellar emission $H_{\lambda}$, reddened SSP spectra are applied. Finally, the photometric and spectroscopic total fluxes of J1203+1624 are fitted with this SED model $\left(F_{\lambda}=R_{\lambda}+H_{\lambda}+B_{\lambda}\right)$.

Fitting results suggest that the AGN emission is deeply buried in dust with a color excess $A_{K}=5.83 \pm 0.25$ if the SMC extinction law is assumed. This is consistent with a significantly red WISE color of $W_{1}-W_{4}=8.48,2.42 \mathrm{mag}$ higher than that of the quasar composite. At this level of high extinction, the transmitted spectrum is negligible even in the NIR, which suggests that J1203+1624 is a Seyfert 2 galaxy. We checked the line profile of the hydrogen line with the largest wavelength in our spectrum, i.e., $\mathrm{Pa} \alpha$, which is dominated by the NEL component (right end of upper panel of Figure 1), consistent with the SED modeling result of no obvious transmitted BEL emission in the NIR. Since that the NELR is almost free from reddening (Figure 2), the obscurer should be located between the NELR and BELR and is very likely the dusty torus presumed by the AGN unification models. After extinction correction, the luminosity of this buried nucleus is found to be $L_{\mathrm{bol}}=5.7 \pm 1.2 \times 10^{45} \mathrm{erg} \mathrm{s}^{-1}$. This estimated bolometric luminosity is $\sim 1000$ times that of $L_{[\mathrm{O} \text { III] } \lambda 5007}$ (Table 1), similar to the sample mean ratio of 3400 ( $1 \sigma$ range of $\sim 1000-10,000$ ) for the 58 bright quasars at lowto-intermediate redshift (Pennell et al. 2017). The blue nuclear emission in the UV is only $\sim 3.6 \% \pm 0.3 \%$ of the reddened AGN radiation. Such a weak blue nuclear emission is also discovered in the high-polarization reddened quasar OI 287 (Goodrich \& Miller 1988; Li et al. 2015) and the heavily obscured quasar SDSS J0006+1215 (Zhang et al. 2017a), where it is interpreted as scattered AGN radiation. To find out 


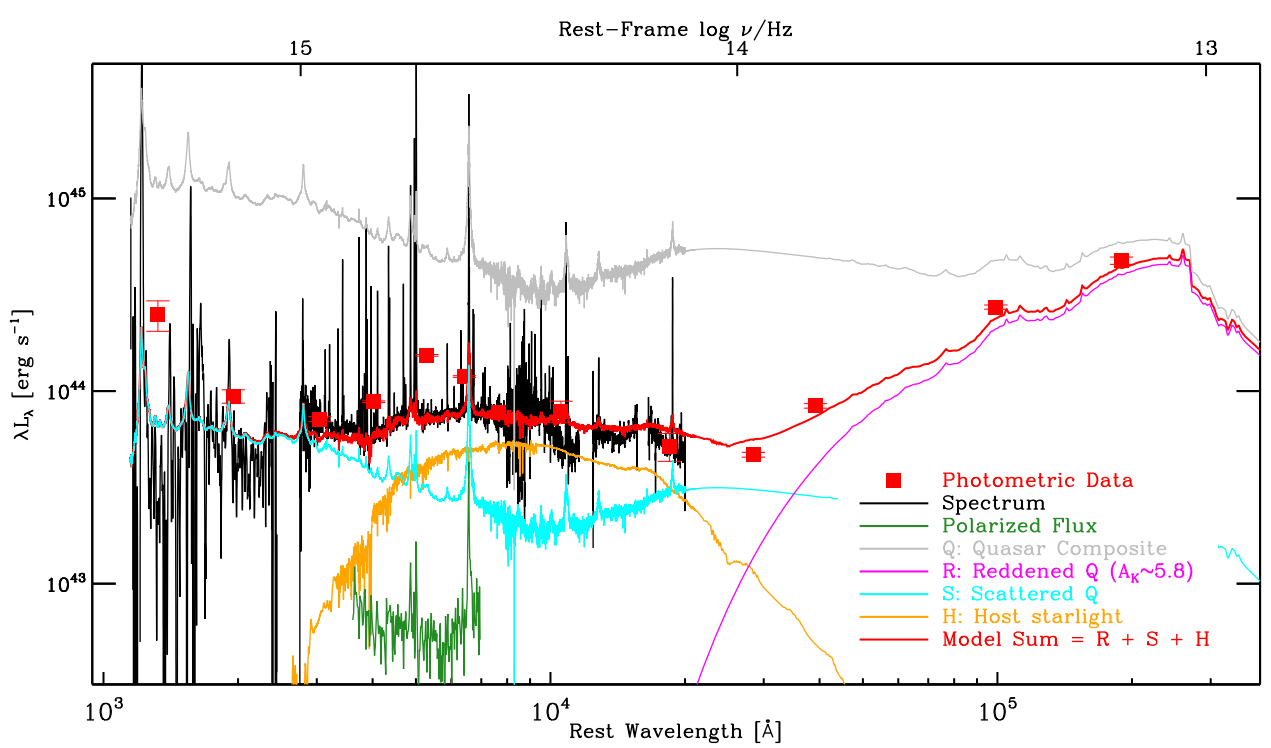

Figure 4. SED modeling of J1203+1624. The black line is the combined spectrum, and red squares are the photometric data. They are fitted with three components: a reddened quasar composite (magenta line), which dominates in the MIR; the starlight of the host galaxy (orange line), which contributes significantly in the IR-optical; and a scattered quasar composite (cyan line), which dominates in the UV-optical. The best-fit model is plotted with a red line, and the polarized flux is presented with a green line.

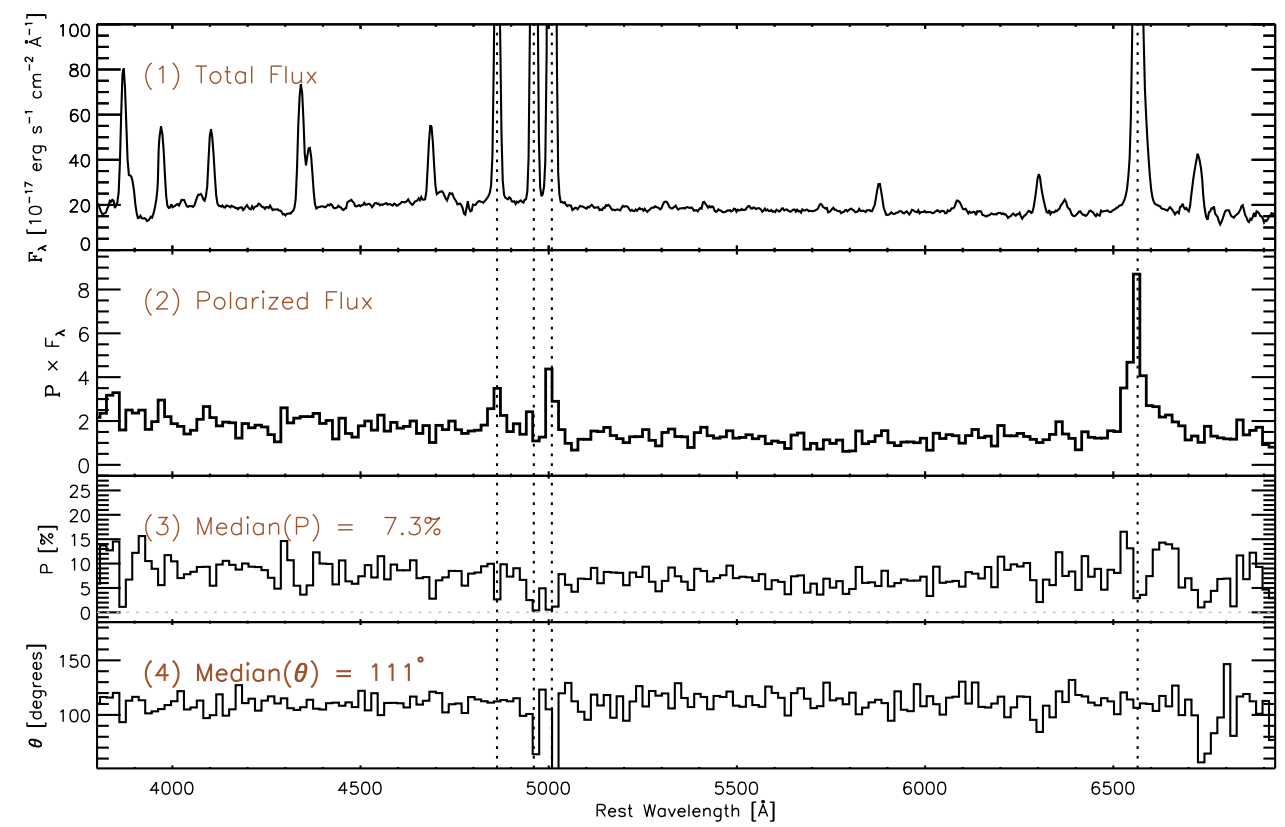

Figure 5. Total and polarized flux spectra $\left(P \times F_{\lambda}\right)$ of J1203+1624 are shown in panels (a) and (b), respectively. In panel (c), the degree of polarization $(P)$ is shown as a function of wavelength, with a median value of $7.3 \%$. The polarization angle $(\theta)$ is displayed in panel (d), which is around $111^{\circ}$ for both continuum and BELs. The data in this figure are binned every 5 pixels.

whether reflection also plays a role in $\mathrm{J} 1203+1624$, we performed spectropolarimetric observations with MMT/SPOL.

\subsection{Scattered AGN Radiation}

In previous works, spectropolarimetry plays an important role in revealing reflected nuclear emission in type 2 AGNs, such as NGC 1068 (Antonucci \& Miller 1985), Mrk 477, Mrk 1210, NGC 7212, and Was 49b (Tran et al. 1992). These observations are among the key motivations of the AGN unification model. Enlightened by these works, it is realized that the buried NLS2 nuclear can possibly be probed and identified via spectropolarimetry of scattered light. Previous spectropolarimetric studies of NLS1s do find significant scattered nuclear radiation in several NLS1s, like Mrk 766, Mrk 1239, etc. (detailed sample studies can be found in Goodrich 1989; Robinson et al. 2011), which suggests that scattering can be common in high-accreting AGNs like NLS1s.

A degree of polarization of $P \sim 7.3 \%$ (Figure 5(c)) is found in the SPOL data of J1203+1624, higher than $90 \%$ of type II AGNs reported in Miller \& Goodrich (1990), Zakamska et al. (2005), and Ramos Almeida et al. (2016), indicating that a significant portion of the optical light comes from scattering. Besides, the $\mathrm{H} \alpha$ and $\mathrm{H} \beta$ BELs are significantly detected in the polarized spectrum $\left(P \times F_{\lambda}\right.$; Figure 5(b)). And, a universal polarized angle of $\theta \sim 111^{\circ}$ for both continuum and BEL 


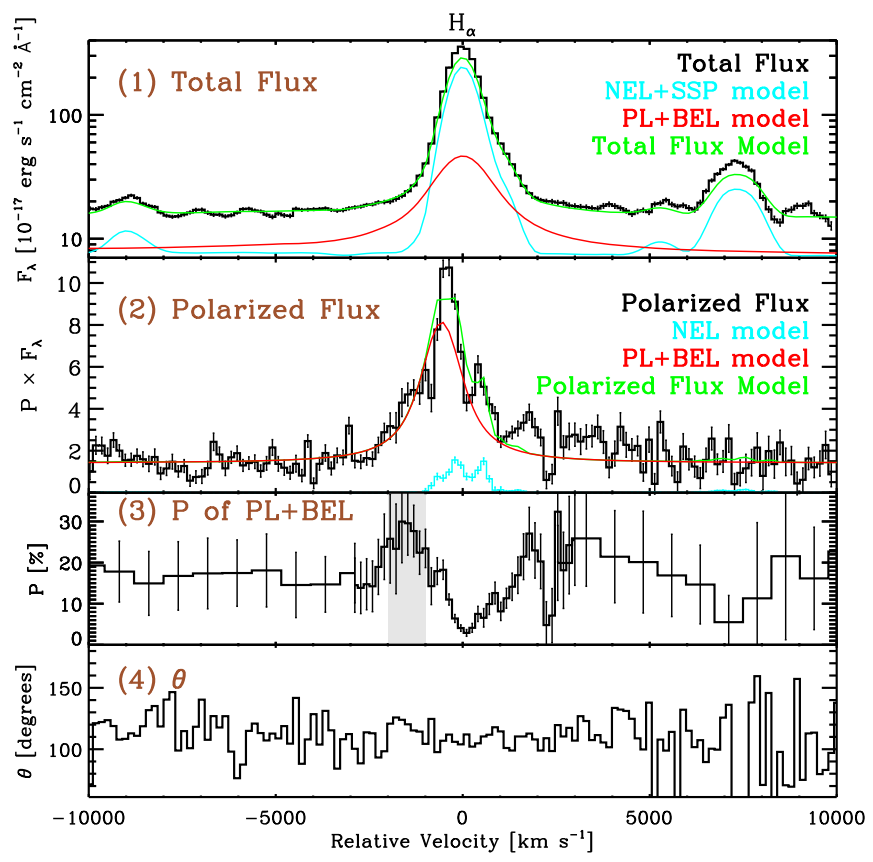

Figure 6. Total and polarized flux spectra $\left(P \times F_{\lambda}\right)$ of $\mathrm{J} 1203+1624$ around the $\mathrm{H} \alpha$ region in velocity space are shown in panels (a) and (b), respectively. The models of NEL(+SSP), PL+BEL, and their sum are shown in cyan, red, and green, respectively, for both the total flux and polarized flux spectrum. After the decomposition, the degree of polarization $P$ for the PL+BEL component is obtained in panel (c); notice that the data at $|v|>3000 \mathrm{~km} \mathrm{~s}^{-1}$ are binned every 5 pixels. Notice the enhanced polarization in the blue wing $\left(v \in[-2000,-1000] \mathrm{km} \mathrm{s}^{-1}\right.$; gray shaded region) of $\mathrm{H} \alpha$. The polarization angle is displayed in panel (d).

(Figure 5(d)) suggests a similar origin for them (similar to 3C 234, reported in Antonucci 1984), while the NELs have a lower $P$ and different polarized angle, indicating a different origin of polarization. Since the polarized radiation from the core components (PL continuum and BEL) comes from a similar origin, we follow Antonucci (1984) in decomposing them from both the total flux and the polarized flux spectrum to analyze the property of its polarization. After being convolved at a resolution of $\sim 7 \AA$, the spectral model (green dotted line in Figure 1; see Section 3 for a detailed description) roughly reproduces the total flux spectrum observed by MMT/SPOL (Figure 6(a)). Similarly, the convolved AGN PL+BEL model (red line) and NEL+SSP model (cyan line) are also obtained. It is assumed that there is no polarization in starlight, since it is much more extended as compared to the nucleus or AGN polar scatters. This leaves us with three components to consider in the polarized flux: AGN PL continuum, BELs, and NELs. Since the strongest isolated NEL in the polarized flux spectrum is $[\mathrm{O}$ III $] \lambda 5007$, we apply its profile in generating the polarized NEL model. On the other hand, as dust extinction seems to be negligible in the NELR (Figure 2) and no apparent wavelength dependence is seen in $P$, electron scattering is assumed here. The NEL flux ratios derived in the total flux spectrum (Table 1) are also applied in obtaining the polarized NEL model (cyan line in Figure 6(b)). After subtracting the polarized NEL model in the polarized flux spectrum, the residual is fitted with a PL and a Lorentzian BEL (red line in Figure 6(b)) in the $\mathrm{H} \alpha$ region. The width of the polarized BEL is found to be $\mathrm{FWHM}_{\text {Polarized BEL }}=1183 \pm 77 \mathrm{~km} \mathrm{~s}^{-1}$, and the line core is blueshifted at $563 \pm 59 \mathrm{~km} \mathrm{~s}^{-1}$. In fact, the emitting regions of $\mathrm{H} \alpha$ and the [O III] $\lambda 5007$ NELs can be quite different
(Baskin \& Laor 2005), and the assumption that $P$ and the profile of polarized $\mathrm{H} \alpha$ and [O III] $\lambda 5007$ NEL are the same can be invalid. Nevertheless, this assumption provides us with chances to reasonably estimate the polarized NELs. After subtracting SSP and NELs, the PL+BEL components in both total flux spectrum and polarized flux spectrum are obtained. The degree of polarization, $P$, of the PL + BEL component is then obtained (Figure 6(c)).

In type 2 AGNs like $\mathrm{J} 1203+1624$, polar scattering is the simplest and best explanation of the polarized nuclear emission. Similarly, the AGN PL continuum and BELs have relatively high $P$ and close polarization angles; they are probably scattered by clouds that posit in similar polar directions relative to the AGN. Since there does not seem to be a dramatic rise at the blue end of the polarized flux spectrum in J1203+1624, electron scattering is favored. ${ }^{11}$ As mentioned earlier, the SED modeling results in a flux ratio of $\sim 3.6 \%$ between the blue AGN and the buried AGN components, which indicates a similar value for the covering fraction of the scattering clouds if the bulk of the blue AGN flux comes from scattering. By assuming the incident line emission of the scattering cloud to be monochromatic, i.e., the polarized BEL is entirely broadened by the thermal motion of electrons, the upper limit of electron temperature is then $T_{\max }=\frac{m_{e} \mathrm{FWHM}_{\text {polarizedBEL }}^{2}}{16 \mathrm{k} \ln 2}=$ $8330 \mathrm{~K}$ (Miller et al. 1991). The temperature of ions/atoms/ molecules is even lower. In order to keep electron scattering over dust scattering at such a low temperature, the scattering cloud should be metal-poor as compared to normal interstellar medium (ISM) to make it dust-deficient. At such a considerable covering fraction and a relatively close distance to the AGN, the scattering clouds should themselves produce emission lines. Since both the scattering clouds and the strong NELR of J1203 +1624 reside in the ionization cone and appear to be metalpoor \& dust-deficient, they are plausibly associated with each other. On the other hand, the $P$ of AGN PL+BEL is higher in the blue wing of the BEL, as shown in Figure 6(c). In the velocity range of $v \in[-2000,-1000] \mathrm{km} \mathrm{s}^{-1}$ around $\mathrm{H} \alpha$ (gray shaded region in Figure 6(c)), the median $(P)$ of PL + BEL is $27.7 \%$, twice the median value for the continuum. Such enhanced $P$ values in the blue wing of the $\mathrm{H} \alpha$ BEL suggests that there should be additional scattering clouds relatively close to the BELR (Smith et al. 2000, 2003). The redshifted polarized BEL can be caused by the inflowing motion of the scattering clouds toward the black hole at the velocity $w=u /\left(1-\cos \langle\phi\rangle_{p}\right) \gtrsim 500 \mathrm{~km} \mathrm{~s}^{-1}$, where $u=$ $563 \pm 59 \mathrm{kms}^{-1}$ is the shifting speed of the polarized emission line (see Section 4.2) and $\langle\phi\rangle_{p}$ is the average scattering angle. Being even closer to the illuminant, this additional part of the scatterer can be highly ionized inflows inside the sublimation radius, which could give rise to the prominent coronal NELs (Rose et al. 2015) in J1203+1624.

\subsection{The Black Hole Parameters}

As a brief summary, the analysis of the SED and polarized flux spectrum strongly suggests that $\mathrm{J} 1203+1624$ is a type 2 AGN whose NLS1 nuclear emission is revealed in scattered light. Based on SED modeling, the bolometric luminosity of $\mathrm{J} 1203+1624$ is $L_{\mathrm{bol}}=5.7 \pm 1.2 \times 10^{45} \mathrm{erg} \mathrm{s}^{-1}$, and the flux ratio between the blue $\mathrm{AGN}$ and the buried nucleus is

\footnotetext{
11 The dust-scattering cross-section rises sharply at short wavelengths (Mathis et al. 1977).
} 
$\sim 3.6 \% \pm 0.3 \%$ (Section 3). Applying this flux ratio to the observed $\mathrm{H} \beta$ BEL, the intrinsic $\mathrm{H} \beta$ BEL of J1203+1624 can be over twice the flux of [O III] $\lambda 5007$, quite normal now among NLS1s. The BEL line widths measured in the total flux spectrum $\left(\mathrm{FWHM}_{\mathrm{BEL}}=1270 \pm 18\right)$ and polarized flux spectrum $\left(\mathrm{FWHM}_{\text {Polarized BEL }}=1183 \pm 77 \mathrm{~km} \mathrm{~s}^{-1}\right)$ are similar to each other. However, these observed BELs originated from either scattering or leaked BEL radiation viewed near the equatorial plane. The velocity width measurement in J1203 +1624 is thus different from the measurement in type 1 AGNs, since in either case, the thermal motion of the scattering electrons or the rotational motion of the disk-like BELR can broaden the observed line width. Therefore, the BEL width we measured can only be treated as an upper limit. Based on the line width and corrected luminosity of the $\mathrm{H} \alpha$ BEL, we apply the empirical relation of Greene \& Ho (2005) and obtain the upper limit of the black hole mass of $\mathrm{J} 1203+1624$, M. $<2.9 \times 10^{7} M_{\odot}$. A lower limit of a super-Eddington rate is obtained, $L_{\mathrm{bol}} / L_{\mathrm{Edd}}>1.5$.

\section{Host Galaxy}

As an NLS2, the active nucleus in J1203+1624 is deeply buried in dust, and the relatively weak starlight from the host galaxy is clearly viewed. A reasonable fit of the broadband data in Section 4 is obtained with a single SSP at an age of $1.4 \mathrm{Gyr}$. At a stellar mass of $M_{*} \sim 7.7 \times 10^{9} M_{\odot}$, the luminosity of starlight, $L_{*} \sim 1.4 \times 10^{10} L_{\odot}$, is only $\sim 1 \%$ of the bolometric luminosity of the dereddened NLS1 nucleus. The SDSS image of J1203+1624 is PSF-dominated; i.e., the spatial extent of the host galaxy is small considering the relatively low redshift of $z=0.1656$. The stellar velocity dispersion is found to be $\sigma_{*}=99 \pm 31 \mathrm{~km} \mathrm{~s}^{-1}$. Following the parameterized $M_{\bullet}-\sigma_{*}$ relation (Tremaine et al. 2002), the predicted black hole mass according to $\sigma_{*}=99 \pm 31 \mathrm{~km} \mathrm{~s}^{-1}$ is $M \bullet^{\prime}=0.80_{-0.58}^{+1.57} \times$ $10^{7} M_{\odot}$, consistent with the upper limit estimated using the $\mathrm{H} \alpha$ BEL.

\section{Conclusion and Prospect}

In this paper, we report the discovery of an unusual NLS1, J1203+1624, with extremely strong NELs. The $\mathrm{EW}_{[\mathrm{O} \text { III }]} \lambda 5007=$ $583 \pm 16 \AA$ is among the top values, even among type 2 quasars. After detailed analysis of its SED, J1203+1624 is found to be a type $2 \mathrm{AGN}$. The nuclear emission is revealed through scattered or possibly leaked radiation in the optical band. The scattered or possibly leaked NLS1 nucleus is identified in the total flux spectrum (FWHM $\mathrm{BEL} \sim 1270 \mathrm{~km} \mathrm{~s}^{-1}$ ) with an Fe II relative strength of $R_{4570} \sim 0.25$ and confirmed in the polarized flux spectrum $\left(\mathrm{FWHM}_{\text {Polarized BEL }} \sim 1183 \mathrm{~km} \mathrm{~s}^{-1}\right.$ ) with a high polarization degree $(P \sim 7.3 \%)$. And the obscurer is at a scale similar to the dusty torus proposed in the unification model. Taking advantage of its heavy extinction and prominent scattering of the core, both the AGN and the host galaxy can be well explored. The black hole mass is estimated to be $M_{0}<$ $2.9 \times 10^{7} M_{\odot}$, with a super-Eddington ratio of $L_{\text {bol }} / L_{\text {Edd }}>1.5$. A mid-aged SSP at an age of $\sim 1.4 \mathrm{Gyr}$ is found, with a stellar mass of $M_{*} \sim 7.7 \times 10^{9} M_{\odot}$ and a stellar velocity dispersion of $\sigma_{*}=99 \pm 31 \mathrm{~km} \mathrm{~s}^{-1}$. A comparison of the nucleus and host galaxy suggests that $\mathrm{J} 1203+1624$ largely follows the $M_{\bullet}-\sigma_{*}$ correlation. Other interesting facts about J1203+1624, like the peculiar NEL system, the inflowing scattering cloud, and the $\mathrm{X}$-ray properties (obscuration, scattering, FeK emission line, and variability) of this optically identified NLS2, are worth detailed follow-up observations. A detailed modeling of the NELR and scattering region of $\mathrm{J} 1203+1624$ based on their tight association is needed to interpret the interesting facts being observed. An interesting subclass of AGNs with obscured SMBHs of small mass accreting at a high rate is represented by J1203+1624. Their existence suggests that NLS1s follow the AGN unification scheme. In addition, the peculiar SED behavior, heavy extinction, and scattering in type 2 AGNs enables detailed exploration of both the active nucleus and the host galaxy. A sample of $\mathrm{J} 1203+1624$ analogs is important for understanding the SMBH-galaxy correlation and their coevolution for NLS1s, which will be conducted in our future work.

We appreciate the detailed and helpful comments from the referee, Robert R. J. Antonucci, which greatly improved the quality of this paper. We thank Victor Manuel Patino Alvarez for suggestions about English writing and some helpful comments. This work is supported by the National Natural Science Foundation of China (NSFC-11473025, 11573024, 11421303) and National Basic Research Program of China (the 973 Program 2013CB834905). H.L., X.P., and L.S. are supported by Natural Science Foundation of Anhui (1808085MA24). D.X. is supported by National Natural Science Foundation of China (NSFC-11773036). P.J. is supported by the National Natural Science Foundation of China (NSFC-11233002). We acknowledge the use of the Multiple Mirror Telescope at Fred Lawrence Whipple Observatory and the Hale 200 inch Telescope at Palomar Observatory through the Telescope Access Program (TAP), as well as the archival data from the GALEX, SDSS, 2MASS, and WISE Surveys. TAP is funded by the Strategic Priority Research Program, Emergence of Cosmological Structures (XDB09000000), National Astronomical Observatories, Chinese Academy of Sciences, and the Special Fund for Astronomy from the Ministry of Finance. Observations obtained with the Hale Telescope were obtained as part of an agreement between the National Astronomical Observatories, Chinese Academy of Sciences, and the California Institute of Technology. Funding for SDSS-III has been provided by the Alfred P. Sloan Foundation, the Participating Institutions, the National Science Foundation, and the U.S. Department of Energy Office of Science. The SDSS-III website is http:// www.sdss3.org/.

\section{ORCID iDs}

Xiang Pan (1D https://orcid.org/0000-0002-6565-2339

S. Komossa (ib https://orcid.org/0000-0002-9214-4428

Shaohua Zhang (iD https://orcid.org/0000-0001-8485-2814

Peng Jiang (10) https://orcid.org/0000-0002-5387-7952

Ning Jiang (i) https://orcid.org/0000-0002-7152-3621

Hongyan Zhou (D) https://orcid.org/0000-0003-1956-9021

\section{References}

Antonucci, R. 1993, ARA\&A, 31, 473

Antonucci, R. R. J. 1984, ApJ, 278, 499

Antonucci, R. R. J., Kinney, A. L., \& Ford, H. C. 1989, ApJ, 342, 64

Antonucci, R. R. J., \& Miller, J. S. 1985, ApJ, 297, 621

Baldwin, J. A., Phillips, M. M., \& Terlevich, R. 1981, PASP, 93, 5

Baskin, A., \& Laor, A. 2005, MNRAS, 358, 1043

Bruzual, G., \& Charlot, S. 2003, MNRAS, 344, 1000

Dewangan, G. C., \& Griffiths, R. E. 2005, ApJL, 625, L31

Dong, X.-B., Zhou, H.-Y., Wang, T.-G., et al. 2005, ApJ, 620, 62 
Fitzpatrick, E. L., \& Massa, D. 2007, ApJ, 663, 320

Gelbord, J. M., Mullaney, J. R., \& Ward, M. J. 2009, MNRAS, 397, 172

Goodrich, R. W. 1989, ApJ, 342, 224

Goodrich, R. W., \& Miller, J. S. 1988, ApJ, 331, 332

Greene, J. E., \& Ho, L. C. 2005, ApJ, 630, 122

Grier, C. J., Peterson, B. M., Horne, K., et al. 2013, ApJ, 764, 47

Jiang, P., Zhou, H., Ji, T., et al. 2013, AJ, 145, 157

Kauffmann, G., Heckman, T. M., Tremonti, C., et al. 2003, MNRAS, 346, 1055

Kewley, L. J., Dopita, M. A., Sutherland, R. S., Heisler, C. A., \& Trevena, J. 2001, ApJ, 556, 121

Komossa, S. 2008, RMxAC, 32, 86

Komossa, S., Voges, W., Xu, D., et al. 2006, AJ, 132, 531

Leighly, K. M. 1999a, ApJS, 125, 297

Leighly, K. M. 1999b, ApJS, 125, 317

Li, Y.-R., Wang, J.-M., Ho, L. C., Du, P., \& Bai, J.-M. 2013, ApJ, 779, 110

Li, Z., Zhou, H., Hao, L., et al. 2015, ApJ, 812, 99

Liu, X.-W., Storey, P. J., Barlow, M. J., et al. 2000, MNRAS, 312, 585

Martin, N., Maurice, E., \& Lequeux, J. 1989, A\&A, 215, 219

Mathis, J. S., Rumpl, W., \& Nordsieck, K. H. 1977, ApJ, 217, 425

Miller, J. S., \& Goodrich, R. W. 1990, ApJ, 355, 456

Miller, J. S., Goodrich, R. W., \& Mathews, W. G. 1991, ApJ, 378, 47

Morrissey, P., Conrow, T., Barlow, T. A., et al. 2007, ApJS, 173, 682

Nagar, N. M., Oliva, E., Marconi, A., \& Maiolino, R. 2002, A\&A, 391, L21

Osterbrock, D. E. 1981, ApJ, 249, 462

Osterbrock, D. E., \& Ferland, G. J. 2006, Astrophysics of Gaseous Nebulae and Active Galactic Nuclei (2nd ed.; Sausalito, CA: Univ. Science Books)

Osterbrock, D. E., \& Pogge, R. W. 1985, ApJ, 297, 166

Pennell, A., Runnoe, J. C., \& Brotherton, M. S. 2017, MNRAS, 468, 1433

Peterson, B. M. 1997, An Introduction to Active Galactic Nuclei (Cambridge: Cambridge Univ. Press)

Pogge, R. W. 2000, NewAR, 44, 381

Ramos Almeida, C., Martínez González, M. J., Asensio Ramos, A., et al. 2016, MNRAS, 461, 1387

Ramos Almeida, C., Pérez García, A. M., Acosta-Pulido, J. A., \& González-Martín, O. 2008, ApJL, 680, L17
Rashed, Y. E., Eckart, A., Valencia-S, M., et al. 2015, MNRAS, 454, 2918

Robinson, A., Young, S., Axon, D. J., \& Smith, J. E. 2011, in ASP Conf. Ser 449, Astronomical Polarimetry 2008: Science from Small to Large Telescopes, ed. P. Bastien et al. (San Francisco, CA: ASP), 431

Rokaki, E., Lawrence, A., Economou, F., \& Mastichiadis, A. 2003, MNRAS, 340, 1298

Rose, M., Elvis, M., \& Tadhunter, C. N. 2015, MNRAS, 448, 2900

Schlafly, E. F., \& Finkbeiner, D. P. 2011, ApJ, 737, 103

Schmidt, G. D., Stockman, H. S., \& Smith, P. S. 1992, ApJL, 398, L57

Schmidt, G. D., Weymann, R. J., \& Foltz, C. B. 1989, PASP, 101, 713

Skrutskie, M. F., Cutri, R. M., Stiening, R., et al. 2006, AJ, 131, 1163

Smith, P. S., Schmidt, G. D., Hines, D. C., Cutri, R. M., \& Nelson, B. O. 2000, ApJL, 545, L19

Smith, P. S., Schmidt, G. D., Hines, D. C., \& Foltz, C. B. 2003, ApJ, 593, 676

Tarchi, A., Castangia, P., Columbano, A., Panessa, F., \& Braatz, J. A. 2011, A\&A, 532, A125

Tran, H. D., Miller, J. S., \& Kay, L. E. 1992, ApJ, 397, 452

Tremaine, S., Gebhardt, K., Bender, R., et al. 2002, ApJ, 574, 740

Vanden Berk, D. E., Richards, G. T., Bauer, A., et al. 2001, AJ, 122, 549

Véron-Cetty, M.-P., Joly, M., \& Véron, P. 2004, A\&A, 417, 515

Véron-Cetty, M.-P., Véron, P., \& Gonçalves, A. C. 2001, A\&A, 372, 730

Wills, B. J., \& Browne, I. W. A. 1986, ApJ, 302, 56

Wright, E. L., Eisenhardt, P. R. M., Mainzer, A. K., et al. 2010, AJ, 140, 1868

York, D. G., Adelman, J., Anderson, J. E., Jr., et al. 2000, AJ, 120, 1579

Yuan, W., Zhou, H. Y., Komossa, S., et al. 2008, ApJ, 685, 801

Zafar, T., Møller, P., Watson, D., et al. 2015, A\&A, 584, A100

Zakamska, N. L., Schmidt, G. D., Smith, P. S., et al. 2005, AJ, 129, 1212

Zakamska, N. L., Strauss, M. A., Krolik, J. H., et al. 2003, AJ, 126, 2125

Zhang, S., Zhou, H., Shi, X., et al. 2017a, ApJ, 836, 86

Zhang, S., Zhou, H., Shi, X., et al. 2017b, ApJ, 845, 126

Zhou, H., Ge, J., Lu, H., et al. 2010, ApJ, 708, 742

Zhou, H., Wang, T., Yuan, W., et al. 2006, ApJS, 166, 128

Zhou, H.-Y., Wang, T.-G., Zhou, Y.-Y., Cheng, L., \& Dong, X.-B. 2002, ApJ, 581,96 